\title{
Effects of Direct Switching Dual Bronchodilators between Dry Powder and Soft Mist Inhalers in COPD Patients
}

\author{
Taisuke Akamatsu, Toshihiro Shirai, Hiromasa Nakayasu, Kanami Tamura, Toshihiro Masuda, \\ Shingo Takahashi, Yuko Tanaka, Hirofumi Watanabe, Yutaro Kishimoto, Kyohei Oishi, \\ Mika Saigusa, Akito Yamamoto, Satoru Morita, Kazuhiro Asada
}

Department of Respiratory Medicine, Shizuoka General Hospital, Shizuoka, Japan

Email: toshihiro-shirai@i.shizuoka-pho.jp

\begin{abstract}
How to cite this paper: Akamatsu, T., Shirai, T., Nakayasu, H., Tamura, K., Masuda, T., Takahashi, S., Tanaka, Y., Watanabe, H., Kishimoto, Y., Oishi, K., Saigusa, M., Yamamoto, A., Morita, S. and Asada, K. (2020) Effects of Direct Switching Dual Bronchodilators between Dry Powder and Soft Mist Inhalers in COPD Patients. Open Journal of Respiratory Diseases, 10, 11-19. https://doi.org/10.4236/ojrd.2020.101002
\end{abstract}

Received: December 27, 2019

Accepted: February 7, 2020

Published: February 10, 2020

Copyright $\odot 2020$ by author(s) and Scientific Research Publishing Inc. This work is licensed under the Creative Commons Attribution International License (CC BY 4.0).

http://creativecommons.org/licenses/by/4.0/

(c) (i) Open Access

\begin{abstract}
Objective: Dual bronchodilation with long-acting muscarinic antagonist and long-acting $\beta 2$-agonist combinations are available worldwide in COPD patients. However, the choice of agents remains under debate. We hypothesized that switching devices between dry powder and soft mist inhalers without a wash-out period to mimic clinical practice would improve clinical symptoms and lung function. The aim of this study was to examine the effects of switching between once-daily glycopyrronium/indacaterol (GLY/IND) or umeclidinium/vilanterol (UMEC/VI), dry powder inhalers, and tiotropium/olodaterol (TIO/OLO), a soft mist inhaler, in COPD patients. Methods: This was a prospective, open-label, 8-week, observational study with follow-up. Subjects included 57 COPD patients, who attended outpatient clinics at Shizuoka General Hospital for routine check-ups between February and December 2015, receiving GLY/IND (50/110 $\mu \mathrm{g})$ or UMEC/VI (62.5/25 $\mu \mathrm{g})$. After an 8-week run-in period, medications were switched to TIO/OLO $(5 / 5 \mu \mathrm{g})$. Study outcomes included patient's global rating (PGR), modified MRC (mMRC), COPD assessment test (CAT), and spirometric and forced oscillatory parameters after 8 weeks. PGR used in this study was a 7-point scale ranging from 1 to 7 , with 4 in the middle. Patients who consented to switch from TIO/OLO to GLY/IND or UMEC/VI were followed-up thereafter. Results: In total, 53 patients completed the study (mean age, 75 years; 48 males and 5 females; GOLD $1 / 2 / 3 / 4=19 / 27 / 6 / 1 ; \operatorname{mMRC} 0 / 1 / 2 / 3 / 4=$ 14/22/12/4/1; UMEC/VI 26, GLY/IND 27). PGR, mMRC, and CAT improved in $20(38 \%), 9(17 \%)$, and 15 patients (28\%), respectively. Respiratory system resistance at $5 \mathrm{~Hz}(\mathrm{R} 5), 20 \mathrm{~Hz}(\mathrm{R} 20)$, and the difference between R5 and R20
\end{abstract}


(R5 - R20) significantly improved. In a follow-up of 16 patients after switching from TIO/OLO to UMEC/VI (9) or GLY/IND (7), PGR, mMRC, and CAT improved in 5 (31\%), 3 (12\%), and 4 patients (25\%), respectively, and R20 significantly improved $(\mathrm{p}=0.011)$. Conclusions: Switching dual bronchodilators between dry powder and soft mist inhalers improves symptoms and airway narrowing in some COPD patients.

\section{Keywords}

Device, Dry Powder Inhaler, Dual Bronchodilator, Soft Mist Inhaler, Switching

\section{Introduction}

Combination treatment with a long-acting muscarinic antagonist (LAMA) and long-acting $\beta 2$-agonist (LABA) increases forced expiratory volume in $1 \mathrm{~s}$ (FEV1) and reduces symptoms compared to monotherapy [1]. There are 3 combinations of a LAMA and LABA in a single inhaler available in Japan, including once-daily glycopyrronium/indacaterol (GLY/IND) and umeclidinium/vilanterol (UMEC/VI), dry powder inhalers, and tiotropium/olodaterol (TIO/OLO), a soft mist inhaler, in order of approval. There is a meta-analysis of dual bronchodilation with LAMA/LABA [2]; however, the choice of agents remains under debate. In clinical practice, clinicians sometimes experience the improvement of symptoms and lung function after switching medications and devices. The recent GOLD documents recommend that inhaler technique and adherence should be assessed following the implementation of therapy and state that switching inhaler devices is an option for adjustments in pharmacological treatment [1]. However, to our knowledge, there has been no study assessing the effectiveness of switching medication and devices. Thus, we hypothesized that switching devices between dry powder and soft mist inhalers without a wash-out period to mimic clinical practice would improve clinical symptoms and lung function in COPD patients. The aim of this study was to examine the effects of switching between dry powder inhalers and a soft mist inhaler in a real-world setting.

\section{Methods}

\subsection{Subjects}

Outpatients with COPD at Shizuoka General Hospital, seen between February and December 2015, were enrolled in this study. All patients fulfilled the definition of GOLD, and the COPD grading was based on the GOLD classification [1]. The inclusion criteria were as follows: 1) age over 40 years, and 2) receiving GLY/IND (50/110 $\mu \mathrm{g} /$ day) or UMEC/VI (62.5/25 $\mu \mathrm{g} /$ day). Exclusion criteria were as follows: patients who: 1) had asthma, or 2) had exacerbation of COPD in the 4 weeks preceding the study. 


\subsection{Study Design}

This was a prospective, single-arm, open-label, 8-week, observational study (Figure 1). At the first visit, after 8 weeks of a run-in period, medications were switched to TIO/OLO (5/5 $\mu \mathrm{g} /$ day) without a washout period. The study doctors, nurses, or pharmacists checked the inhaler technique of each patient during the run-in period and after switching. Study outcomes after 8 weeks included patient's global rating (PGR), modified Medical Research Council (mMRC), COPD assessment test (CAT), spirometry, and forced oscillation technique (FOT). Patients who consented to switch from TIO/OLO to GLY/IND or UMEC/VI were followed-up thereafter (Figure 2).

This study was conducted in accordance with the principles of the Declaration of Helsinki, this protocol was approved by the Institutional Review Board of Shizuoka General Hospital, and written informed consent was obtained from all patients prior to the study.

\subsection{Measurements}

\section{PGR}

PGR was evaluated by a 7-point scale ranging from 1 to 7 (1, very much better; 2 , much better; 3 , a little better; 4 , no change; 5 , a little worse; 6 , much worse; 7 , very much worse).

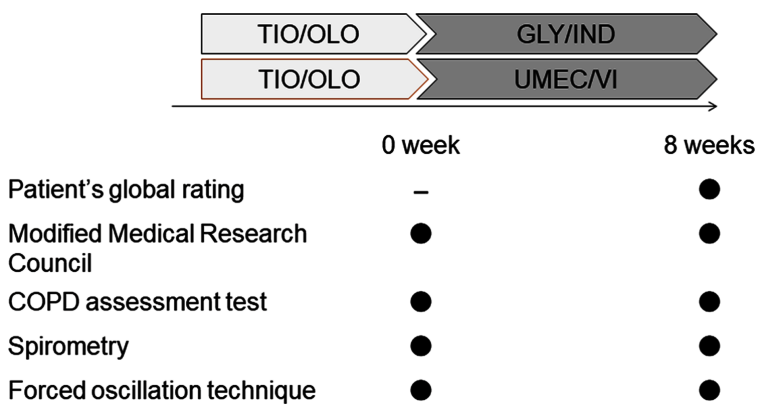

Figure 1. Study protocol 1. GLY/IND or UMEC/VI was switched to TIO/OLO. Abbreviations: GLY-IND, glycopyrronium/indacaterol; TIO/OLO, tiotropium/olodaterol; UMEC/VI, umeclidinium/vilanterol.

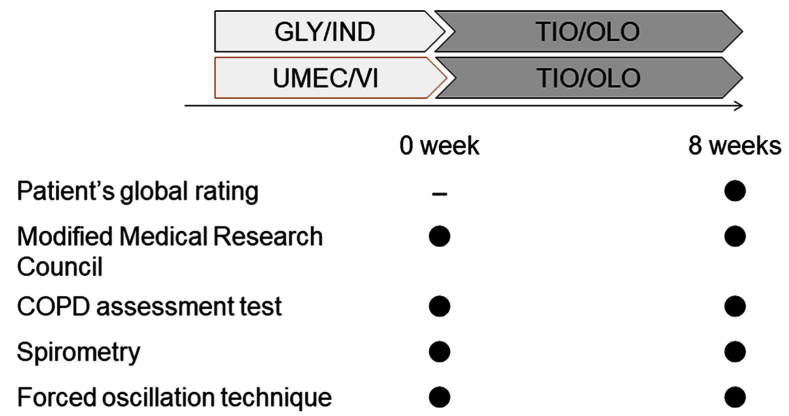

Figure 2. Study protocol 2. TIO/OLO was switched to GLY/IND or UMEC/VI. Abbreviations: GLY-IND, glycopyrronium/indacaterol; TIO/OLO, tiotropium/olodaterol; UMEC/VI, umeclidinium/vilanterol. 


\section{Assessment of symptoms}

The mMRC score was used to evaluate dyspnea in daily living, grading from 0 (only get breathless with strenuous exercise) to 4 (too breathless to leave the house or breathless when dressing) [3].

The CAT (Japanese version, supplied by GlaxoSmithKline, Japan) consists of eight items (cough, phlegm, chest tightness, breathlessness going up hills/stairs, activity limitations at home, confidence leaving home, sleep, and energy) that assess and quantify the symptoms and the impact of COPD [4]. Each item is scored from 0 to 5 , giving a total score range from 0 to 40 , corresponding to the best and worst health statuses, respectively. The minimal clinically important difference was 2 points.

FOT and spirometry

Measurement of FOT and spirometry were performed in that order to avoid any influence of forced expiration. Broadband frequency FOT was performed using the MostGraph-01 (Chest M.I. Co. Ltd., Tokyo, Japan) in accordance with standard recommendations [5]. We used respiratory system resistance (Rrs) at 5 and $20 \mathrm{~Hz}$ (R5 and R20, respectively) and the difference between R5 and R20 (R5 - R20) as an indicator of the frequency dependence of Rrs. We also used and respiratory system reactance (Xrs) at $5 \mathrm{~Hz}$ (X5), which reflects the elastic and inertial properties of the lung, resonant frequency (Fres) where Xrs crosses zero and the elastic and inertial forces are opposite and equal in magnitude, and a low-frequency reactance area (AX), which is an integral of Xrs at $5 \mathrm{~Hz}$ to Fres. Oscillatory indices were expressed as mean values during a respiratory cycle. Furthermore, the difference between inspiratory and expiratory phases $(\Delta)$ of X5 was also used as a marker of expiratory flow limitation [6]. All measured data were expressed as post-bronchodilator values.

Spirometry was performed using computerized equipment (model CHESTAC-8800; Chest M.I. Co. Ltd., Tokyo, Japan) in accordance with the American Thoracic Society (ATS)/European Respiratory Society (ERS) guidelines for spirometry [7] while patients were on daily medications. Measured data were expressed as post-bronchodilator values. Predicted values for pulmonary function tests were obtained from the Japanese Respiratory Society guidelines [8].

\subsection{Statistical Analyses}

The quantitative data were summarized as means with standard deviations and paired $t$-tests were used to compare changes between weeks 0 and 8 . The statistical significance for all analyses was set at $p<0.05$ (two-tailed). All analyses were performed using EZR version 1.27 (Saitama Medical Center, Jichi Medical University, Saitama, Japan) [9].

\section{Results}

The patient flow and the clinical characteristics of the subjects at baseline are shown in Figure 3 and Table 1. The mean age of the subjects was 75 years and 


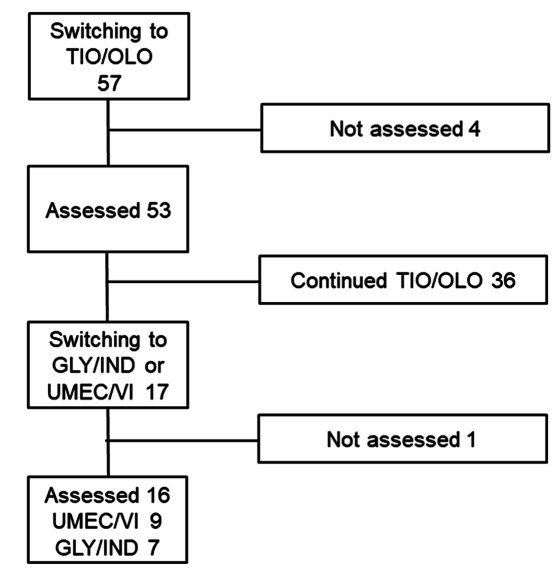

Figure 3. Patient flow. Abbreviations: GLY-IND, glycopyrronium/indacaterol; TIO/OLO, tiotropium/olodaterol; UMEC/VI, umeclidinium/vilanterol.

Table 1. Clinical characteristics of the subjects (switching to TIO/OLO).

\begin{tabular}{cc}
\hline & $\mathrm{n}=53$ \\
\hline Age, yr & $75 \pm 7$ \\
Gender, male/female, $\mathrm{n}$ & $48 / 5$ \\
Smoking history, current/ex/never & $0 / 51 / 2$ \\
Pack-years & $52.9 \pm 27.1$ \\
GOLD, 1/2/3/4, n & $19 / 27 / 6 / 1$ \\
mMRC, 0/1/2/3/4, n & $14 / 22 / 12 / 4 / 1$ \\
CAT score & $9.9 \pm 8.1$ \\
UMEC-VI/GLY-IND, n & $26 / 27$ \\
FVC, L & $3.22 \pm 0.75$ \\
FEV1, L & $1.87 \pm 0.60$ \\
\% FEV1, \% predicted & $74.2 \pm 23.8$ \\
IC & $2.15 \pm 0.58$ \\
\hline
\end{tabular}

Values are shown as mean \pm SD or numbers. Abbreviations: CAT, COPD assessment test; FEV1, forced expiratory volume in $1 \mathrm{~s}$; FVC, forced vital capacity; GLY-IND, glycopyrronium/indacaterol; GOLD, global initiative for chronic obstructive lung disease; IC, inspiratory capacity; mMRC, modified medical research council; TIO/OLO, tiotropium/olodaterol; UMEC/VI, umeclidinium/vilanterol.

there was male-dominance. Most of the subjects were ex-smokers, while there were two never smokers. The mean pack-years was 52.9. Overall, the subjects had mild or moderate airflow limitation based on the GOLD classification. Two-thirds of the subjects had mild dyspnea (mMRC 0 and 1), while the mean CAT score was around 10. The mean FEV1 was $1.87 \mathrm{~L}$ (74.2\% predicted). Of the 57 patients, 53 completed the first switching and were assessed. Two patients dropped out and the remaining two patients developed exacerbation. PGR, mMRC, and CAT improved in 20 (38\%), 9 (17\%), and 15 patients (28\%), respectively. The mean R5, R20, and R5 - R20 significantly improved; however, there was no improvement in other parameters (Table 2). In 21 patients who had 
Table 2. Changes in clinical parameters after 8 weeks (switching to TIO/OLO).

\begin{tabular}{cccc}
\hline & Before switching & After switching & $p$-value \\
\hline mMRC & $1.16 \pm 0.98$ & $1.06 \pm 0.79$ & 0.083 \\
CAT & $9.9 \pm 8.1$ & $9.8 \pm 7.9$ & 0.940 \\
FVC, L & $3.22 \pm 0.75$ & $3.23 \pm 0.73$ & 0.885 \\
FEV1, L & $1.87 \pm 0.60$ & $1.89 \pm 0.64$ & 0.352 \\
IC, $\mathrm{L}$ & $2.15 \pm 0.58$ & $2.12 \pm 0.56$ & 0.517 \\
$\mathrm{R} 5, \mathrm{cmH}_{2} \mathrm{O} / \mathrm{L} / \mathrm{s}$ & $3.29 \pm 1.34$ & $3.00 \pm 1.10$ & 0.026 \\
$\mathrm{R} 20, \mathrm{cmH}_{2} \mathrm{O} / \mathrm{L} / \mathrm{s}$ & $2.48 \pm 0.90$ & $2.29 \pm 0.72$ & 0.045 \\
$\mathrm{R} 5-\mathrm{R} 20, \mathrm{cmH}_{2} \mathrm{O} / \mathrm{L} / \mathrm{s}$ & $0.81 \pm 0.50$ & $0.71 \pm 0.46$ & 0.030 \\
$\mathrm{X} 5, \mathrm{cmH}_{2} \mathrm{O} / \mathrm{L} / \mathrm{s}$ & $-1.01 \pm 1.11$ & $-0.84 \pm 0.91$ & 0.058 \\
$\Delta \mathrm{X} 5, \mathrm{cmH}_{2} \mathrm{O} / \mathrm{L} / \mathrm{s}$ & $0.60 \pm 1.47$ & $0.36 \pm 0.98$ & 0.072 \\
$\mathrm{Fres}_{\mathrm{H}} \mathrm{Hz}$ & $10.86 \pm 4.67$ & $10.29 \pm 4.71$ & 0.100 \\
$\mathrm{ALX}, \mathrm{cmH}_{2} \mathrm{O} / \mathrm{L} / \mathrm{s} \mathrm{x} \mathrm{Hz}$ & $6.67 \pm 10.02$ & $5.24 \pm 7.77$ & 0.096 \\
\hline
\end{tabular}

Values are shown as mean \pm SD. Abbreviations: ALX, low-frequency reactance area; CAT, COPD assessment test; $\Delta$, difference between inspiratory and expiratory phases; FEV1, forced expiratory volume in $1 \mathrm{~s}$; Fres, resonant frequency; IC, inspiratory capacity; mMRC, modified medical research council; R5 and R20, respiratory system resistance at 5 and $20 \mathrm{~Hz}$, respectively; TIO/OLO, tiotropium/olodaterol; X5, respiratory system reactance at $5 \mathrm{~Hz}$.

CAT score $\geq 10$, PGR improved in 7 patients $(33 \%)$ and the mean mMRC improved significantly $(1.95 \pm 0.86$ to $1.67 \pm 0.73, \mathrm{p}=0.010)$. However, there was no improvement in other parameters.

Of the 53 patients, 17 consented to switch from TIO/OLO to UMEC/OLO or GLY/IND and 16 completed the second switching (Figure 3). One patient dropped out. Table 3 shows the clinical characteristics. PGR, mMRC, and CAT improved in 5 (31\%), 3 (12\%), and 4 patients (25\%), respectively. The mean R20 significantly improved; however, there was no improvement in other parameters (Table 4).

\section{Discussion}

We prospectively assessed the effects of switching between dry powder inhalers and a soft mist inhaler in COPD patients in a real-world setting. The results revealed that the patient reported outcomes, including PGR, mMRC, and CAT, and objective FOT parameters improved after switching from dry powder to soft mist inhalers. Furthermore, similar improvement was observed after subsequent switching from soft mist to dry powder inhalers albeit in fewer patients.

A previous meta-analysis of indirect comparison between fixed-dose LAMA/LABA combinations suggested that there might be a gradient of effectiveness between GLY/IND, UMEC/VI, and TIO/OLO in trough FEV1 but not in dyspnea index [2]. Subsequently, the direct head-to-head comparison of UMEC/VI and TIO/OLO for 8 weeks with an interim 3-week washout found that trough FEV1 was significantly higher in UMEC/VI treatment than in 
Table 3. Clinical characteristics of the subjects (switching from TIO/OLO).

\begin{tabular}{cc}
\hline & $\mathrm{n}=16$ \\
\hline Age, yr & $75 \pm 8$ \\
Gender, male/female, $\mathrm{n}$ & $15 / 1$ \\
Smoking history, current/ex/never & $0 / 16 / 0$ \\
Pack-years & $65.2 \pm 26.0$ \\
GOLD, 1/2/3/4, n & $6 / 7 / 2 / 1$ \\
mMRC, 0/1/2/3/4, n & $5 / 7 / 2 / 1 / 1$ \\
CAT score & $10.1 \pm 10.2$ \\
UMEC-VI/GLY-IND, n & $9 / 7$ \\
FVC, L & $3.55 \pm 0.77$ \\
FEV1, L & $1.99 \pm 0.70$ \\
\% FEV1, \% predicted & $72.3 \pm 23.5$ \\
IC & $2.33 \pm 0.58$ \\
\hline
\end{tabular}

Values are shown as mean \pm SD or numbers. Abbreviations: CAT, COPD assessment test; FEV1, forced expiratory volume in $1 \mathrm{~s}$; FVC, forced vital capacity; GLY-IND, glycopyrronium/indacaterol; GOLD, global initiative for chronic obstructive lung disease; IC, inspiratory capacity; mMRC, modified medical research council; TIO/OLO, tiotropium/olodaterol; UMEC/VI, umeclidinium/vilanterol.

Table 4. Changes in clinical parameters after 8 weeks (switching from TIO/OLO).

\begin{tabular}{cccc}
\hline & Before switching & After switching & $p$-value \\
\hline mMRC & $1.13 \pm 1.15$ & $1.13 \pm 1.02$ & 1.000 \\
CAT & $10.1 \pm 10.2$ & $9.6 \pm 10.1$ & 0.240 \\
FVC, $\mathrm{L}$ & $3.55 \pm 0.77$ & $3.56 \pm 0.81$ & 0.847 \\
FEV1, $\mathrm{L}$ & $1.99 \pm 0.70$ & $2.00 \pm 0.69$ & 0.712 \\
$\mathrm{IC}, \mathrm{L}$ & $2.33 \pm 0.58$ & $2.35 \pm 0.71$ & 0.846 \\
$\mathrm{R} 5, \mathrm{cmH}_{2} \mathrm{O} / \mathrm{L} / \mathrm{s}$ & $2.82 \pm 0.95$ & $2.97 \pm 1.06$ & 0.125 \\
$\mathrm{R} 20, \mathrm{cmH}_{2} \mathrm{O} / \mathrm{L} / \mathrm{s}$ & $2.11 \pm 0.49$ & $2.29 \pm 0.58$ & 0.011 \\
$\mathrm{R} 5-\mathrm{R} 20, \mathrm{cmH}_{2} \mathrm{O} / \mathrm{L} / \mathrm{s}$ & $0.71 \pm 0.51$ & $0.68 \pm 0.56$ & 0.600 \\
$\mathrm{X} 5, \mathrm{cmH}_{2} \mathrm{O} / \mathrm{L} / \mathrm{s}$ & $-1.00 \pm 1.18$ & $-0.84 \pm 0.84$ & 0.361 \\
$\Delta \mathrm{X} 5, \mathrm{cmH}_{2} \mathrm{O} / \mathrm{L} / \mathrm{s}$ & $0.71 \pm 1.32$ & $0.51 \pm 0.80$ & 0.400 \\
$\mathrm{Fres}_{\mathrm{H}} \mathrm{Hz}$ & $10.63 \pm 5.80$ & $10.72 \pm 5.73$ & 0.432 \\
$\mathrm{ALX}, \mathrm{cmH} \mathrm{H}_{2} \mathrm{O} / \mathrm{L} / \mathrm{s} \mathrm{x} \mathrm{Hz}$ & $6.92 \pm 9.93$ & $5.69 \pm 7.08$ & 0.096 \\
\hline
\end{tabular}

Values are shown as mean \pm SD. Abbreviations: ALX, low-frequency reactance area; CAT, COPD assessment test; $\Delta$, difference between inspiratory and expiratory phases; FEV1, forced expiratory volume in $1 \mathrm{~s}$; Fres, resonant frequency; IC, inspiratory capacity; mMRC, modified medical research council; R5 and R20, respiratory system resistance at 5 and $20 \mathrm{~Hz}$, respectively; TIO/OLO, tiotropium/olodaterol; X5, respiratory system reactance at $5 \mathrm{~Hz}$.

TIO/OLO treatment [10]. The results of this study cannot be compared simply with the previous reports. However, we found that switching GLY/IND or UMEC/VI to TIO/OLO was useful in some patients since subjective improve- 
ment was confirmed. The improvement even after switching from TIO/OLO to GLY/IND or UMEC/VI suggests that this may be associated with devices used, characteristics, or changes in adherence.

There is a difference in the devices used between GLY/IND and UMEC/VI: the former uses Breezhaler and the latter Ellipta. Concerning the efficacy, the previous indirect meta-analysis reported that there was no difference in trough FEV1, dyspnea index, or quality of life between the two medications [2].

The improvement in the FOT parameters but not in FEV1 highlights the difference between the FOT and spirometry. The FOT is a lung function test to detect airway narrowing during tidal breathing that does not require forced expiration, as in spirometry [6]. Since the changes in FOT parameters are generally greater than those observed in spirometry, the significant changes in the FOT parameters, but not in FEV1, were supposed to be obtained in this study. Basically, Rrs parameters, including R5, R20, and R5 - R20, are thought to reflect airway caliber [6].

There are limitations in this study. GLY/IND and UMEC/VI were assessed together as dry powder inhalers although there are differences in the device and pharmacological characteristics between the two combinations. However, both combinations had been widely used before TIO/OLO combinations became available in Japan, thereby enabling the comparison between dry powder inhalers and a soft mist inhaler. When switching the devices, a wash-out period was not set to mimic clinical practice. This may lead to the usefulness of switching LAMA/LABA combinations when response to treatment is inappropriate.

There was also an improvement in symptoms and R20 after switching from TIO/OLO to UMEC/OLO or GLY/IND. From a theoretical point of view, symptoms as well as lung function parameters can be worse after switching back to the initial inhalers. One possible explanation might be a carrying over effect of $\mathrm{TIO} / \mathrm{OLO}$ in addition to the improvement of adherence.

\section{Conclusion}

In conclusion, switching dual bronchodilators between dry powder and soft mist inhalers improves symptoms and airway narrowing in some COPD patients.

\section{Acknowledgements}

There was no funding in this study.

\section{Conflicts of Interest}

The authors declare no conflicts of interest regarding the publication of this paper.

\section{References}

[1] Global Initiative for Chronic Obstructive Lung Disease (2019) Global Strategy for the Diagnosis, Management, and Prevention of Chronic Obstructive Pulmonary 
Disease. https://goldcopd.org

[2] Calzetta, L., Rogliani, P., Matera, M.G. and Cazzola, M. (2016) A Systematic Review with Meta-Analysis of Dual Bronchodilation with LAMA/LABA for the Treatment of Stable COPD. Chest, 149, 1181-1196. https://doi.org/10.1016/j.chest.2016.02.646

[3] Bestall, J.C., Paul, E.A., Garrod, R., Garnham, R., Jones, P.W. and Wedzicha, J.A. (1999) Usefulness of the Medical Research Council (MRC) Dyspnoea Scale as a Measure of Disability in Patients with Chronic Obstructive Pulmonary Disease. Thorax, 54, 581-586. https://doi.org/10.1136/thx.54.7.581

[4] Jones, P.W., Harding, G., Berry, P., Wiklund, I., Chen, W.H. and Kline, L.N. (2009) Development and First Validation of the COPD Assessment Test. European Respiratory Journal, 34, 648-654. https://doi.org/10.1183/09031936.00102509

[5] Oostveen, E., MacLeod, D., Lorino, H., Farre, R., Hantos, Z., Desager, K., et al. (2003) The Forced Oscillation Technique in Clinical Practice: Methodology, Recommendations and Future Developments. European Respiratory Journal, 22, 1026-1041. https://doi.org/10.1183/09031936.03.00089403

[6] Shirai, T. and Kurosawa, H. (2016) Clinical Application of the Forced Oscillation Technique. Internal Medicine, 55, 559-566. https://doi.org/10.2169/internalmedicine.55.5876

[7] Miller, M.R., Hankinson, J., Brusasco, V., Burgos, F., Casaburi, R., Coates, A., et al. (2005) Standardisation of Spirometry. European Respiratory Journal, 26, 319-338. https://doi.org/10.1183/09031936.05.00034805

[8] The Committee of Pulmonary Physiology, Japanese Respiratory Society (2004) Guidelines for pulmonary function tests. Spirometry, Flow-Volume Curve, Diffusing Capacity of the Lung. Japanese Respiratory Society, Tokyo.

[9] Kanda, Y. (2013) Investigation of the Freely Available Easy-to-Use Software 'EZR' for Medical Statistics. Bone Marrow Transplantation, 48, 452-458. https://doi.org/10.1038/bmt.2012.244

[10] Feldman, G.J., Sousa, A.R., Lipson, D.A., Tombs, L., Barnes, N., Riley, J.H., et al. (2017) Comparative Efficacy of Once-Daily Umeclidinium/Vilanterol and Tiotropium/Olodaterol Therapy in Symptomatic Chronic Obstructive Pulmonary Disease: A Randomized Study. Advances in Therapy, 34, 2518-2533. https://doi.org/10.1007/s12325-017-0626-4 